\title{
ORIGINAL
}

\section{METODOLOGÍA DEL ESTUDIO DE COHORTES DEL PROYECTO ITINERE SOBRE CONSUMIDORES DE HEROÍNA EN TRES CIUDADES ESPAÑOLAS Y CARACTERÍSTICAS BÁSICAS DE LOS PARTICIPANTES}

\author{
Luis de la Fuente de Hoz (1,2), M Teresa Brugal Puig (3), Rosario Ballesta Gómez (4), María J \\ Bravo Portela (1), Gregorio Barrio Anta (5), Antonia Domingo Salvany (6), Teresa Silva do Rosa- \\ rio (2), Mireia Ambrós Hortensi (3) y Grupo del proyecto ITÍNERE (7).
}

(1) Secretaría del Plan Nacional sobre el Sida. Ministerio de Sanidad y Consumo. Madrid

(2) Centro Nacional de Epidemiología. Instituto de Salud Carlos III. Madrid.

(3) Agència de Salut Pública de Barcelona

(4) Fundación Andaluza para la Atención a las Drogodependencias. Sevilla.

(5) Departamento de Medicina Preventiva, Salud Pública e Historia de la Ciencia. Universidad Complutense de Madrid

(6) Unidad de Investigación en Servicios Sanitarios. Institut Municipal d'Investigació Mèdica (IMIM).

(7) El Grupo del Proyecto Itínere está constituido por los autores citados anteriormente más: Sylvina Bassani, Francisco Bru, Carlos Toro, David Fernández, Francisco González, Victoria Jiménez, Daniel Lacasa, Angels Llorens, Eusebio Mejías, Montserrat Neira, José Pulido, Luis Royuela, Sofía Ruiz, Fernando Sánchez, Vicente Soriano, Fernando Vallejo y Nuria Vallés.

\section{RESUMEN}

Fundamento: Los estudios de cohortes permiten monitorizar el impacto del uso de drogas sobre la salud, e identificar los factores condicionantes. El objetivo de este trabajo es describir la metodología y las características básicas de una cohorte de consumidores de heroína diseñada con este fin.

Métodos: Participaron 991 jóvenes consumidores de heroína, seleccionados en la comunidad en Barcelona, Madrid y Sevilla, principalmente mediante nominación de otros participantes $(39,7 \%)$ o de consumidores o exconsumidores no participantes $(44,7 \%)$. Se administró un cuestionario con ordenador y se recogió una muestra de sangre en papel secante. También se registraron sus medidas antropométricas. Se remuneró a participantes y captadores. Se usaron métodos estadísticos uni y bivariados.

Resultados: Un 42,4\% había cambiado alguna vez de vía principal de administración de heroína, sobre todo hacia la inyección en Barcelona y hacia la vía pulmonar en Sevilla. Un 75,8\% (Barcelona), $49,8 \%$ (Madrid), y $15,5 \%$ (Sevilla) se habían inyectado drogas en los últimos 12 meses. En Madrid y Sevilla un 96\%-97\% consumían la heroína sólo en forma de base, y en Barcelona predominaba la heroína-clorhidrato. Frecuentemente mezclaban heroína y cocaína en la misma dosis (generalmente cocaína-base en Madrid y Sevilla, y cocaína-clorhidrato en Barcelona).

Correspondencia:

Luis de la Fuente

Centro Nacional de Epidemiología. Instituto de Salud Carlos III

C/Sinesio Delgado 6

28029 Madrid. Correo Electrónico: lfuente@isciii.es
ABSTRACT

\section{Cohort Study Methodology of the ITINERE Project on Heroin Users in Three Spanish Cities and Main Characteristics of the Participants}

Background: Cohort studies make it possible to monitor the health impact of drug use and to identify related factors. We describe the methodology and baseline characteristics of a cohort of heroin users designed with this objective.

Methods: The participants were 991 young, community-recruited heroin users in Barcelona, Madrid and Seville. Most subjects were named by other participants $(39.7 \%)$ or by non-participating drug users or ex-users $(44.7 \%)$. A computer-aided questionnaire was administered (self-administered with audio for questions related with sex). A dried-blood spot sample was collected and anthropometric measurements were made. Both participants and recruiters received remuneration. Univariate and bivariate statistical methods were used.

Financiación: La fuente principal de financiación del estudio fue la Fundación para Investigación y la Prevención del Sida en España, a través del proyecto FIPSE 3035/99. También se han recibido apoyos para investigaciones específicas de: Redes Temáticas de Investigación Cooperativa (C03-09 y G03-005), Fondo de Investigación Sanitaria (FIS 00/1017, FIS 01/0908), y Delegación del Gobierno para el Plan Nacional sobre Drogas. 
Conclusiones: Persisten importantes diferencias geográficas en la prevalencia de inyección de drogas y en los patrones de consumo de heroína y cocaína, lo que podría explicar la desigual distribución de algunos problemas de salud. Las dificultades para reunir la muestra prevista sugieren un descenso importante de la incidencia de consumo de heroína.

Palabras clave: Estudio de cohortes. Consumidores de heroína. Entrevista asistida por ordenador.
Results: Some $42.4 \%$ had changed the main route of heroin administration, mainly to injection in Barcelona and to the pulmonary route in Seville. About $75.8 \%$ (Barcelona), $49.8 \%$ (Madrid), and $15.5 \%$ (Seville) had injected drugs in the last 12 months. In Madrid and Seville, 96-97\% used heroin in base form, while in Barcelona heroin hydrochloride predominated. Heroin and cocaine were frequently mixed in the same dose (generally base cocaine in Madrid and Seville, and cocaine hydrochloride in Barcelona).

Conclusions: Important geographic differences persist in the prevalence of drug injection and in the patterns of heroin and cocaine use, which could explain the unequal distribution of some health problems. The difficulties encountered in recruiting the sample suggest that the incidence of heroin use has declined considerably.

Key words: Cohort study. Heroin users. Street recruitment. Computer Assisted Personal Interview.

\section{INTRODUCCIÓN}

El Proyecto Itínere pretende integrar varias cohortes de jóvenes usuarios de drogas captados en la comunidad en varias áreas españolas (en principio, Madrid, Barcelona y Sevilla) para evaluar el impacto del uso de drogas sobre la salud y la calidad de vida, su evolución, y los factores condicionantes. Sus resultados deberían permitir evaluar las políticas preventivas con vistas a mejorar su efectividad y eficiencia, e identificar precozmente grupos de alto riesgo que precisan intervención rápida. En este sentido, los estudios de cohortes tienen ventajas porque permiten estimar la incidencia de un evento (inyección de drogas, infección, etc.), una medida más sensible que la prevalencia para monitorizar los cambios, y evaluar el efecto de ciertos factores. Además, al interpretar las asociaciones, se puede asegurar que estos factores preceden en el tiempo al evento.

Actualmente Itinere incluye una cohorte constituida de usuarios de heroína y otra de usuarios de cocaína en fase de captación. Este trabajo se centra en la primera. En España se justifica un estudio de cohortes de usuarios de heroína porque esta droga ha generado en los últimos 25 años un volumen importante de problemas sanitarios graves, sobre todo intoxicaciones agudas (sobredosis), e infecciones por los virus del sida (VIH), y de la hepatitis B (VHB) y C (VHC). Estos problemas han descendido en la última década, pero siguen siendo relevantes, por lo que se precisa un estudio más detallado de los factores condicionantes. En este sentido, la mortalidad por sobredosis ha descendido menos de lo esperado (estimándose en España en el 2000 aproximadamente mil muertes por esta causa ${ }^{1}$ ), y muchos usuarios de heroína siguen precisando atención urgente por sobredosis no mortales ${ }^{2-6}$. La incidencia de VIH ligada a inyección de drogas ha descendido mucho ${ }^{6-8}$, pero el número de inyectores infectados (prevalencia) sigue siendo mucho más alto que en otros países europeos, y descenderá despacio al aumentar la supervivencia por los nuevos antiretrovirales. La prevalencia de infección por VHC es altísima, y su tratamiento supone un coste importante para el sistema de sanitario9 ${ }^{9}$ Las conductas de riesgo siguen siendo frecuentes entre los usuarios de heroína ${ }^{10-11}$, pese a los intensos programas preventivos desarrollados, y su aumento podría provocar cambios bruscos en la epidemia de VIH. Este estudio permitirá monitorizar estas conductas, incluso entre los inyectores jóvenes y recientes, que suelen tener alto riesgo de infectarse, sobre todo por VHC y VHB ${ }^{12-13}$, facilitando el desarrollo precoz de programas de control, y la detección de cambios atribuibles a las intervenciones.

Un estudio como éste, que incluye usuarios de heroína por distintas vías de administración, permite también investigar los cambios en la prevalencia e incidencia de inyec- 
ción de drogas, sus determinantes, y sus efectos sobre el riesgo de intoxicación aguda, infecciones y otros eventos. Hay considerar que en España más del $90 \%$ de los inyectores de drogas usan heroína ${ }^{10-16}$, por lo que esta cohorte es muy eficiente para este fin. En este sentido, se sabe que la inyección implica un riesgo de sobredosis mucho más alto que otras vías de uso ${ }^{5}$, y que en España ha descendido mucho el número de inyectores ${ }^{17-18}$, lo que ha sido decisivo para controlar la epidemia de $\mathrm{VIH}^{11}$. Sin embargo, este descenso puede detenerse o revertir en zonas y momentos concretos ${ }^{19}$.

Finalmente, hay otros aspectos hasta ahora poco estudiados que podrían investigarse también en esta cohorte. Por ejemplo: a) Los efectos del uso de cocaína fumada (base), sobre la salud y el riesgo de transmisión de VIH, ya que el uso de cocaína fumada en España se concentra entre los usuarios de heroína ${ }^{6,20-22}$, b) Los problemas de salud mental de los usuarios de heroína y su relación con el abuso de drogas. De hecho, se ha observado peor estado físico y ajuste psicosocial, peor pronóstico del tratamiento de la dependencia, y mayor gravedad del uso de drogas en los usuarios de drogas con trastornos mentales ${ }^{23,24}$, c) El uso de servicios sociosanitarios por los usuarios de heroína, sobre todo los de reducción del daño (mantenimiento con metadona, intercambio de jeringas). Se sabe que el mantenimiento con metadona disminuye mucho el riesgo de sobredosis ${ }^{25}$, e infección por $\mathrm{VIH}^{26,27}$, d) La calidad de vida, y su relación con los patrones de uso de heroína y las intervenciones. Se sabe que la calidad de vida de los usuarios de heroína mejora mucho tras el tratamiento con metadona ${ }^{28,29}$.

El hecho de seleccionar a los participantes en la comunidad permite identificar grupos de alto riesgo que no acuden a los servicios preventivos y asistenciales, y obtener muestras más representativas, con resultados más extrapolables al conjunto de usuarios de heroína. La selección de jóvenes aumenta la eficiencia del estudio para detectar cambios, porque los jóvenes es más probable que inicien nuevas conductas o las cambien. Además, parece más adecuado prevenir las conductas de riesgo en las primeras etapas de consumo. Por último, la selección en varias áreas geográficas permite detectar diferencias, y evaluar, y controlar el efecto de factores ecológicos. En este sentido, la evolución de la vía de uso de heroína en las tres áreas de selección de la cohorte ha sido muy diferente (en Barcelona es aún frecuente inyectársela, mientras que en Sevilla y Madrid predomina la vía pulmonar desde hace tiempo ${ }^{17,30}$ ). Y también han sido bastante diferentes las políticas de reducción de daño, por lo que se puede evaluar el efecto de algunos factores «ecológicos» (tipo de heroína disponible, políticas, etc.) sobre los cambios detectados.

A continuación se describe la metodología y las características basales (sociodemográficas y patrones de uso de drogas) de la cohorte de jóvenes consumidores de heroína del Proyecto Itinere.

\section{SUJETOS Y MÉTODOS}

Los participantes debían cumplir en la visita basal cuatro criterios de inclusión y ninguno de exclusión. Los de inclusión fueron: 1) haber usado heroína al menos 12 días en los 12 meses previos, y al menos un día en los 3 meses previos, 2) tener entre 18 y 30 años (confirmado con DNI), 3) haber residido en el área metropolitana de Madrid, Barcelona o Sevilla al menos 6 de los 12 meses previos, y 4) cumplir varios requisitos administrativos: donar muestra de sangre en papel secante, y mechón de cabello, firmar un consentimiento informado aceptando las condiciones para participar y la no devolución de los resultados analíticos, y autorizando a los investigadores a usar sus datos personales para localizarlo en registros sociosanitarios (mortalidad, morbilidad o instituciones penitenciarias), si se perdiera contacto 
con él, dar datos identificativos para su seguimiento (DNI o pasaporte, domicilio, teléfono, contactos para localizarlo si no asiste a visitas programadas), comprometerse a acudir a dos visitas, uno y dos años tras la basal, y a mantener contacto telefónico con el equipo del proyecto entre las visitas anuales, y firmar un recibí al cobrar las gratificaciones. Los criterios de exclusión fueron: 1) No ser capaz de contestar con fluidez en castellano, y 2) Tener un riesgo muy alto de pérdida en el seguimiento (como prever cambiar de ciudad de residencia el próximo año o ser trabajador del sexo itinerante).

Entre abril de 2001 y diciembre de 2003 se obtuvieron datos basales válidos de 991 consumidores (364 en Barcelona, 427 en Madrid y 200 en Sevilla), todos captados en la comunidad, independientemente de los servicios de tratamiento de drogodependencias. Al no existir un marco muestral adecuado para seleccionarlos, se recurrió a muestreos no probabilísticos. Se intentó que un trabajador en cada ciudad captara directamente a algunos consumidores en diversos escenarios (muestreo dirigido o targeted sampling $)^{31}$, confiando en que ellos nominarían a otros (muestreo en bola de nieve o snowball sampling $)^{32}$. Sin embargo, esto fue poco productivo, optándose por el «muestreo dirigido por el respondente» (respondent driven sampling) ${ }^{33}$ : un sistema de bola de nieve con incentivos por participar y captar o nominar a otros participantes.

La muestra final se reunió con cuatro procedimientos (tabla 1). Para mejorar la representatividad se diversificaron los lugares y las formas de captación, y se buscaron activamente ciertos tipos de consumidores (integrados, muy jóvenes, con poco tiempo de consumo, etc.). Los captadores externos fueron mayoritariamente usuarios de heroína no participantes (por su edad o por otra razón), o exusuarios, que trabajaron de forma irregular por problemas de salud, desaparición, ingreso en prisión, etc. El resto fueron profesionales o personas implicadas en la aten- ción a los consumidores, siendo en general poco productivos. Se les retribuyó con $18 €$ por cada participante captado. La nominación por participantes consistió en pedirles a todos al acabar la visita basal que intentaran captar a otros consumidores seleccionables. Cada nominador solía proponer a 1 ó 2 candidatos, y recibía $10 €$ por cada uno que acudía a la visita basal. No se limitó el número de participantes nominados o procedentes de un captador, por las dificultades de captación. Para captar directamente participantes los trabajadores de campo iban en distintos momentos a escenarios donde era bastante probable encontrar usuarios de heroína. Hacían el cribado a quienes veían o suponían que usaban drogas, y, si eran seleccionables, concertaban la visita o les pedían su teléfono para localizarlos. Los escenarios se identificaron previamente (estudios etnográficos y epidemiológicos, servicios de reducción del daño), o durante el trabajo de campo. Los más productivos fueron las zonas de venta y uso masivo de drogas, como Barranquilla (el más productivo con diferencia) y Pitis en Madrid, Can Tunis, el Bronx de Nou Barris y la Mina en Barcelona, y Polígono Sur, y barrios de Alameda y San Pablo en Sevilla. También fueron bastante productivos los programas de intercambio de jeringuillas (PIJ), centros «calor y café» (como Antaris en Sevilla, o SPOTT en Barcelona), y casas ocupadas (sobre todo en Barcelona), y menos las zonas de prostitución, áreas de reunión o trabajo de consumidores (zonas de aparcacoches clandestinos en Sevilla), albergues, farmacias, etc. Durante la captación no hubo incidentes violentos destacables. Algunos participantes fueron atraídos al estudio por publicidad (tarjetas, trípticos, carteles), distribuida en zonas de consumo y programas de reducción del daño.

\section{Recogida basal de información}

\section{Contacto inicial y visita basal}

Tras comprobar el cumplimiento de los criterios de selección, se informaba al candi- 
Tabla 1

Formas de captación de los participantes

\begin{tabular}{|c|c|c|c|c|c|c|c|c|}
\hline & \multicolumn{2}{|c|}{ Barcelona } & \multicolumn{2}{|c|}{ Madrid } & \multicolumn{2}{|c|}{ Sevilla } & \multicolumn{2}{|c|}{ Total } \\
\hline & $\mathbf{n}$ & $\%$ & $\mathbf{n}$ & $\%$ & $\mathbf{n}$ & $\%$ & $\mathbf{n}$ & $\%$ \\
\hline $\begin{array}{l}\text { Captación directa por los trabajadores de campo del estudio } \\
\text { en diversos escenarios }\end{array}$ & 55 & 15,1 & 90 & 21,1 & 24 & 12,0 & 169 & 17,1 \\
\hline Nominación por participantes & 240 & 65,9 & 184 & 43,1 & 42 & 21,0 & 466 & 47,0 \\
\hline $\begin{array}{l}\text { Trabajo de captadores externos (consumidores, } \\
\text { exconsumidores, profesionales, etc) }\end{array}$ & 68 & 18,7 & 142 & 33,3 & 128 & 64,0 & 338 & 34,1 \\
\hline Participación directa en respuesta a publicidad & 0 & 0,0 & 11 & 2,6 & 6 & 3,0 & 17 & 1,7 \\
\hline TOTAL & 364 & 100,0 & 427 & 100,0 & 200 & 100,0 & 991 & 100,0 \\
\hline
\end{tabular}

dato de las condiciones de participación. Algunos rechazaron participar por no mostrar el DNI o dar los identificadores. A otros les preocupaba que familiares o instituciones (centros de tratamiento, policía) pudiesen conocer su situación; pero generalmente participaron tras explicarles la forma de recogida y protección de datos. Luego se concertaba la visita basal. Aproximadamente el $50 \%$ de los citados no acudieron inicialmente a la misma, aunque a veces se realizó tras tres o cuatro intentos fallidos. En la visita, además de la entrevista, y tomar muestras biológicas y medidas antropométricas, se informó sobre el estudio, subrayando la confidencialidad y la forma de almacenar los datos, se examinó el cumplimiento de los requisitos administrativos, y se informó a demanda sobre los recursos donde el participante podía informarse sobre drogas, VIH, otras infecciones, o a hacerse análisis o tratamientos de la dependencia u otras patologías.

La visita fue realizada por trabajadores entrenados (antropólogos, trabajadores sociales, psicólogos, sociólogos o personal sanitario), generalmente en centros sanitarios o sociales, y raramente en centros de tratamiento de drogodependencias (aclarando que el estudio era ajeno al centro). En Madrid y Barcelona fue difícil conseguir salas de visita, seguramente por el rechazo a esta población. Se dividió en dos bloques de entrevista, con un descanso para tomar muestras y medidas. Duró entre una y 2,5 horas. Durante la misma hubo pocos incidentes desagradables. Se remuneró a cada participante con $18 €$ lo que facilitó la captación, pero atrajo a gente que intentó engañar sobre los criterios de selección.

\section{Entrevista cara-a-cara guiada por ordenador}

Se usó un cuestionario compuesto sobre todo por preguntas cerradas sobre los 12 meses previos a la entrevista, con algunas preguntas abiertas sobre percepciones (Anexo I). Para construir la sección de sobredosis no mortales se hicieron entrevistas en profundidad a 20 consumidores. Además, se hizo un pretest para evaluar la adecuación del cuestionario, y modificar aspectos confusos o mal aceptados por los participantes. La mayoría de las preguntas se administraron mediante entrevista personal guiada por ordenador (Computer Assisted Personal Interview o CAPI $)^{42-44}$. Las preguntas sexuales fueron autoadministradas con versión audio (Audio Computer Administered Self Interview o ACASI) a los que tenían ciertas habilidades lectoras. El cuestionario se programó con Questionnaire Development System (QDS) ${ }^{45}$, que permite evitar pregun- 
tas redundantes, diseñar saltos y filtros automáticos, formular diferencialmente las preguntas por sexo, y chequear inconsistencias. En la entrevista a algunos participantes les costó mantener la atención o concentrarse por estar en síndrome de abstinencia, ansiosos o algo intoxicados. Al final, a menudo estaban cansados.

\section{Toma y análisis de muestras biológicas. Mediciones antropométricas}

Se tomó sangre de la yema del dedo mediante lancetas de un solo uso. Tras indicarle cómo hacerlo de forma segura ${ }^{46}$, el participante se pinchaba, aplicaba la sangre sobre una tira de papel secante, etiquetada con el código de conexión, y echaba los materiales usados a un contenedor. Las tiras de papel se secaban a la sombra durante 24 horas, y luego se enviaban al Hospital Carlos III de Madrid, donde se refrigeraban a $4^{\circ} \mathrm{C}$ hasta su análisis. De las 991 muestras 20 (2\%) no pudieron analizarse. Se determinaron marcadores frente a VIH, VHC, VHB, y HTLV, con los métodos detallados en Bassani et $\mathrm{al}^{47}$. Estos métodos no establecen un diagnóstico clínico preciso, por lo que los participantes que querían saber su estado serológico fueron derivados a centros gratuitos. Igualmente, se tomó una muestra de cabello, que podría servir para validar el autoinforme sobre uso de drogas ${ }^{48}$. La medida objetiva de altura y peso sólo se hizo a un $77 \%$ de los participantes, obteniéndose en los casos restantes (casi todos de Barcelona) por autoinforme.

\section{Almacenamiento y análisis de la información}

Los datos para identificar y localizar a los sujetos se recogieron en fichas de papel, que se destruyeron al grabarlos en una base de datos de gestión computerizada. Esta base se encriptó y se situó en un ordenador diferente al de la base epidemiológica, que incluye el resto de los datos del estudio y no tiene identificadores personales. La conexión entre ambas bases para modificar o agregar datos (visitas de seguimiento, analíticas) se aseguró con un código de conexión. Antes de grabarlos se desecharon algunos cuestionarios por problemas graves de completitud o coherencia de las respuestas. Para obtener los resultados presentados se utilizaron métodos estadísticos uni y bivariados. Para el cálculo de las proporciones se eliminaron del denominador los casos con valores desconocidos. La significación estadística de las diferencias de proporciones se evaluó con la prueba ji cuadrado, rechazándose la hipótesis nula si $\mathrm{p}<0,05$.

\section{RESULTADOS}

Características sociodemográficas: De los 991 participantes, la mayoría eran hombres. Un 32,8\%-53,3\% tenían 25 años o menos, y un 4,5\%-17,6\% habían nacido en el extranjero. Menos de un $4 \%$ habían acabado estudios universitarios. La proporción con estudios secundarios era menor en Sevilla que en Madrid o Barcelona (tabla 2). Para la mayoría las fuentes principales de ingresos eran el trabajo, el robo o venta de drogas, y otras actividades marginales (venta y espectáculos callejeros, mendicidad, recoger chatarra o cartón, vigilar aparcamientos, etc.). Más de la mitad estaban en paro. La mayoría vivían en hogares (casas, apartamentos u otros), siendo muy variable la proporción de personas sin techo o domicilio fijo (43,7\% en Barcelona, y menos de $17 \%$ en Madrid y Sevilla). De los alojados en hogares, en Madrid y Sevilla la mayoría convivían con su familia de origen (padres, hermanos y/o otros familiares). En cambio en Barcelona no había convivientes predominantes, y un $42,3 \%$ convivía con amigos, compañeros u otras personas. Menos de un $7 \%$ vivían solos. Entre un 22\% (Barcelona) y un $41,5 \%$ (Sevilla) tenían algún hijo (tabla 2). En cuanto a las situaciones de conflicto social, un $50,8 \%$ se habían fugado de casa 
Tabla 2

Características sociodemográficas de los jóvenes consumidores de heroína de Barcelona, Madrid y Sevilla, 2001-2003 (\%)

\begin{tabular}{|c|c|c|c|c|c|}
\hline & Barcelona & Madrid & Sevilla & Total & $\mathbf{p}^{s}$ \\
\hline & $(n=364)$ & $(n=427)$ & $(n=200)$ & $(n=991)$ & \\
\hline Hombres & 69,0 & 71,7 & 82,5 & 72,9 & $* *$ \\
\hline Grupo de edad & & & & & $* * *$ \\
\hline$\leq 25$ & 53,3 & 32,8 & 41,5 & 42,1 & \\
\hline$>25$ & 46,7 & 67,2 & 58,5 & 57,9 & \\
\hline Nacido en el extranjero & 17,6 & 8,7 & 4,5 & 10,2 & $* * *$ \\
\hline Nivel de educación aprobado & & & & & * \\
\hline Enseñanza primaria o menos & 34,1 & 41,0 & 76,5 & 45,7 & \\
\hline Enseñanza secundaria & 63,2 & 55,1 & 23,5 & 51,7 & \\
\hline Educación universitaria & 2,7 & 3,8 & 0,0 & 2,6 & \\
\hline Situación laboral durante la mayor parte del tiempo $^{A}$ & & & & & ns \\
\hline Empleo regular & 30,8 & 32,3 & 31,5 & 31,5 & \\
\hline Desempleado & 56,8 & 54,6 & 56,1 & 56,1 & \\
\hline Otra & 12,4 & 13,1 & 12,4 & 12,4 & \\
\hline Fuente principal de ingresos ${ }^{A}$ & & & & & * \\
\hline Trabajo con o sin contrato & 33,8 & 37,9 & 28,5 & 34,5 & \\
\hline Seguro, ayuda social & 3,7 & 3,8 & 1,5 & 3,3 & \\
\hline Dinero de la familia & 4,6 & 5,9 & 3,5 & 4,9 & \\
\hline Prostitución & 4,0 & 4,9 & 3,5 & 4,9 & \\
\hline Robos, venta de drogas & 25,4 & 27,6 & 29,5 & 27,2 & \\
\hline Otras actividades marginales & 28,5 & 19,9 & 33,5 & 25,7 & \\
\hline Tipo de alojamiento en que ha vivido la mayor parte del tiempo ${ }^{\Lambda}$ & & & & & $* * *$ \\
\hline Hogares individuales o familiares & 53,0 & 79,4 & 81,0 & 70,0 & \\
\hline Institución & 3,3 & 6,6 & 2,0 & 4,4 & \\
\hline Sin domicilio fijo/okupas & 29,4 & 7,3 & 10,5 & 16,0 & \\
\hline Sin techo & 14,3 & 6,8 & 6,5 & 9,5 & \\
\hline \multicolumn{6}{|l|}{ Convivientes habituales $^{\mathrm{A}, \mathrm{B}}$} \\
\hline Pareja y/o hijos & 33,9 & 27,9 & 27,9 & 30,0 & ns \\
\hline Padres, hermanos, otros familiares & 45,0 & 72,7 & 68,2 & 62,0 & $* * *$ \\
\hline Amigos, compañeros, otras personas & 42,3 & 17,4 & 10,1 & 32,4 & *** \\
\hline Ninguno, viven solos & 4,9 & 3,6 & 6,1 & 4,6 & ns \\
\hline Hijos & & & & & $* * *$ \\
\hline Ninguno & 78,0 & 73,2 & 58,5 & 72,0 & \\
\hline Uno & 17,9 & 20,4 & 22,5 & 19,9 & \\
\hline Más de uno & 4,1 & 6,3 & 19,0 & 8,1 & \\
\hline Alguna fuga del hogar de más de dos días antes de los 18 años & & & & & ns \\
\hline Sí & 51,1 & 53,2 & 45,0 & 50,8 & \\
\hline No & 48,9 & 46,8 & 55,0 & 49,2 & \\
\hline $\begin{array}{l}\text { Algún ingreso en centro de reforma para menores por cometer } \\
\text { delitos }\end{array}$ & & & & & ns \\
\hline Sí & 12,9 & 10,8 & 7,0 & 10,8 & \\
\hline No & 87,1 & 89,2 & 93,0 & 89,2 & \\
\hline Tiempo total en prisión en su vida & & & & & ns \\
\hline Nunca & 59,3 & 58,1 & 54,0 & 57,7 & \\
\hline$<1$ año & 19,0 & 18,7 & 19,0 & 18,9 & \\
\hline 1 año ó más & 21,7 & 23,2 & 27,0 & 23,4 & \\
\hline
\end{tabular}

\$ Nivel de significación estadística de la comparación entre ciudades, usando ji cuadrado: ns= no significativo; * $\mathrm{p}<0,05 ; * * \mathrm{p}<0,01$ $* * * \mathrm{p}<0.001$

A Referido a los últimos 12 meses. ${ }^{\text {в }}$ Cálculos efectuados sobre el total de los que se alojaron en hogares familiares (casas, apartamentos barrocas, chabolas, caravanas, etc.). Se excluyen por tanto los que se alojaron en establecimientos colectivos como pensiones, hoteles o instituciones, y las personas sin techo o sin hogar. 
Tabla 3

Patrones de consumo de heroína entre los jóvenes consumidores de Barcelona, Madrid y Sevilla. 2001-2003

\begin{tabular}{|c|c|c|c|c|c|}
\hline & Barcelona & Madrid & Sevilla & Total & $\mathbf{p}^{\$}$ \\
\hline & $(n=364)$ & $(n=427)$ & $(n=200)$ & $(n=991)$ & \\
\hline Edad de primer consumo & & & & & $* * *$ \\
\hline$<15$ & 11,5 & 23,7 & 27,0 & 19,9 & \\
\hline $15-16$ & 26,1 & 25,1 & 22,0 & 24,8 & \\
\hline $17-18$ & 29,4 & 24,4 & 28,5 & 27,0 & \\
\hline $19-20$ & 17,9 & 16,2 & 11,0 & 15,7 & \\
\hline$>20$ & 15,1 & 10,8 & 11,5 & 12,5 & \\
\hline Vía de primer consumo & & & & & $* * *$ \\
\hline Fumada & 39,7 & 65,6 & 88,9 & 60,8 & \\
\hline Inyectada & 13,8 & 9,1 & 4,0 & 9,8 & \\
\hline Esnifada en polvo por la nariz & 46,6 & 25,3 & 7,0 & 29,4 & \\
\hline Tiempo entre el primer consumo y el consumo semanal & & & & & $* * *$ \\
\hline$<1$ mes & 22,8 & 30,8 & 43,6 & 30,3 & \\
\hline $1-5$ meses & 31,6 & 26,7 & 32,6 & 29,7 & \\
\hline $6-11$ meses & 10,8 & 8,2 & 8,3 & 9,2 & \\
\hline 12 meses o más & 34,8 & 34,2 & 15,5 & 30,8 & \\
\hline Vía habitual de administración al inicio del consumo semanal & & & & & $* * *$ \\
\hline Fumada & 40,8 & 77,7 & 92,8 & 67,2 & \\
\hline Inyectada & 25,2 & 12,7 & 5,2 & 15,8 & \\
\hline Esnifada en polvo por la nariz & 34,0 & 9,6 & 2,1 & 17,0 & \\
\hline Cambio de vía habitual de consumo alguna vez en la vida & 54,4 & 43,2 & 19,1 & 42,4 & *** \\
\hline Dirección del último cambio de vía & & & & & $* * *$ \\
\hline De inyectada a no-inyectada & 12,3 & 50,6 & 82,8 & 34,3 & \\
\hline De no-inyectada a inyectada & 87,7 & 49,4 & 17,2 & 65,7 & \\
\hline Frecuencia de consumo en últimos 12 meses & & & & & $* * *$ \\
\hline Todos los días & 38,7 & 43,8 & 61,0 & 45,4 & \\
\hline 5-6 días/semana & 8,2 & 14,5 & 8,0 & 10,9 & \\
\hline 3-4 días/semana & 26,1 & 17,8 & 12,5 & 19,8 & \\
\hline 1-2 días/semana o menos & 26,9 & 23,9 & 18,5 & 23,9 & \\
\hline Vía habitual de consumo en últimos 12 meses & & & & & $* * *$ \\
\hline Fumada & 28,3 & 82,9 & 99,0 & 66,1 & \\
\hline Inyectada & 59,3 & 16,9 & 1,0 & 29,3 & \\
\hline Esnifada en polvo por la nariz & 12,4 & 0,2 & 0,0 & 4,6 & \\
\hline $\begin{array}{l}\text { Uso de heroína sola o mezclada con cocaína en la misma dosis en últimos } 12 \\
\text { meses }\end{array}$ & & & & & $* * *$ \\
\hline Siempre mezclada & 7,7 & 4,1 & 53,2 & 14,8 & \\
\hline La mayoría de las veces mezclada & 19,8 & 30,7 & 32,9 & 27,2 & \\
\hline Aproximadamente la mitad de las veces mezclada y la mitad sola & 8,6 & 11,2 & 8,7 & 98,0 & \\
\hline La mayoría de las veces sola & 40,4 & 48,8 & 2,3 & 36,9 & \\
\hline Siempre sola & 23,5 & 5,1 & 2,9 & 11,2 & \\
\hline Tipo de heroína consumida en últimos 12 meses & & & & & $* * *$ \\
\hline Siempre blanca & 56,0 & 0,5 & 0,0 & 20,8 & \\
\hline La mayoría de las veces blanca & 19,5 & 0,0 & 0,0 & 7,2 & \\
\hline Aproximadamente la mitad de las veces blanca y la mitad marrón & 4,9 & 0,2 & 0,5 & 2,0 & \\
\hline La mayoría de las veces marrón & 12,4 & 2,3 & 2,0 & 6,0 & \\
\hline Siempre marrón & 7,1 & 96,9 & 97,5 & 64,0 & \\
\hline
\end{tabular}


Tabla 3 (Cont.)

\begin{tabular}{|c|c|c|c|c|c|}
\hline Lugar de consumo más frecuente en últimos 12 meses & & & & & *** \\
\hline Casas o alojamientos donde ha vivido & 26,6 & 19,4 & 31,0 & 24,4 & \\
\hline Espacios públicos abiertos (calles, plazas, parques, etc.) & 15,1 & 15,7 & 28,5 & 18,1 & \\
\hline Zonas de venta y consumo masivo de drogas & 49,7 & 41,5 & 0,5 & 36,2 & \\
\hline Coches & 1,9 & 14,3 & 8,0 & 8,5 & \\
\hline Otro lugar & 6,6 & 9,1 & 32,0 & 12,8 & \\
\hline Compañeros de consumo más frecuentes & & & & & $* * *$ \\
\hline Ninguno (consume solo habitualmente) & 32,1 & 29,7 & 47,5 & 34,2 & \\
\hline Pareja sexual estable & 15,7 & 22,2 & 10,0 & 17,4 & \\
\hline Amigos muy cercanos que no son pareja sexual & 34,9 & 16,7 & 26,5 & 25,4 & \\
\hline Conocidos casuales que no son pareja sexual & 14,0 & 26,2 & 11,0 & 18,7 & \\
\hline Con otras personas & 3,3 & 4,9 & 5,0 & 4,3 & \\
\hline
\end{tabular}

más de dos días antes de los 18 años, un $10,8 \%$ habían estado en centros de reforma o internamiento para menores, y un $42,3 \%$ en prisión (tabla 2).

Patrones de consumo de heroína: La mayoría iniciaron el uso de heroína con 18 años o menos, generalmente por vía fumada en Sevilla $(88,9 \%)$ y Madrid $(65,6 \%)$, y esnifada $(46,6 \%)$ o fumada $(39,7 \%)$ en Barcelona. El inicio por inyección fue raro (4\%$13,8 \%$ ). La mayoría tardó menos de 6 meses entre el primer uso y el uso semanal. Al iniciarse el uso semanal la vía esnifada había perdido relevancia en favor de la inyectada en Barcelona y de la fumada en Madrid. Entre un $19,1 \%$ (Sevilla) y un $54,4 \%$ (Barcelona) cambiaron alguna vez en la vida de vía habitual (más frecuente) de uso de heroína, considerando sólo los cambios que duraron un mes o más. En Barcelona predominó el cambio de vía no-inyectada a inyectada $(87,7 \%)$, en Sevilla el contrario $(82,2 \%)$ y en Madrid ninguno de los dos (tabla 3).

Con respecto a los patrones de uso reciente (últimos 12 meses), un 45,3\% usaban heroína a diario o casi a diario (5-6 días/semana), y un 23,9\% la usaban 1-2 días/semana. La vía habitual de uso reciente era diferente por ciudad: en Madrid (82,9\%) y Sevilla (99\%) predominaba ampliamente la fumada, y en Barcelona la inyectada
$(59,3 \%)$, aunque existían bastantes fumadores y esnifadores. En Sevilla la heroína se solía usar mezclada con cocaína en la misma dosis $(86,1 \%$ la usaba habitualmente así), mientras que no sucedía lo mismo en Madrid y Barcelona. En cuanto al tipo de heroína, en Madrid y Sevilla más del $96 \%$ usaba siempre heroína-base (marrón) mientras que en Barcelona un $75,5 \%$ usaba habitualmente heroína-clorhidrato (blanca) (tabla 3).

En Madrid y Barcelona los lugares preferentes de uso de heroína eran las zonas de venta y uso masivo de drogas, seguidas de los alojamientos donde vivían, y los espacios abiertos (calles, plazas, parques, etc.). En cambio, en Sevilla apenas se consumía en zonas de venta y uso masivo, prefiriéndose los alojamientos, los espacios abiertos, y otros lugares. La mayoría de los participantes solían usar la heroína con otros, sobre todo amigos cercanos, conocidos o pareja sexual estable. La proporción de los que consumían solos fue mayor en Sevilla que en Barcelona o Madrid (tabla 3).

Prevalencia de uso reciente de otras drogas: En los últimos 12 meses había sido casi universal el uso de tabaco y cocaína, y muchos habían usado la cocaína en forma de base o crack $(78,6 \%)$, o mezclada con heroína en la misma dosis $(>73,9 \%)$. La prevalencia de uso de cocaína base era más alta en 
Tabla 4

Prevalencia de consumo de drogas psicoactivas entre los jóvenes consumidores de Barcelona, Madrid y Sevilla. 2001-2003

\begin{tabular}{|c|c|c|c|c|c|c|c|c|c|c|}
\hline & \multicolumn{4}{|c|}{ Alguna vez en la vida } & \multirow[b]{2}{*}{$p^{s}$} & \multicolumn{4}{|c|}{ Últimos 12 meses } & \multirow[b]{2}{*}{$p^{\$}$} \\
\hline & Barcelona & Madrid & Sevilla & Total & & Barcelona & Madrid & Sevilla & Total & \\
\hline & $(\mathrm{n}=364)$ & $(\mathrm{n}=427)$ & $(\mathrm{n}=200)$ & $(\mathrm{n}=991)$ & & $(n=364)$ & $(\mathrm{n}=427)$ & $(\mathrm{n}=200)$ & $(\mathrm{n}=991)$ & \\
\hline \multicolumn{11}{|l|}{ HEROÍNA } \\
\hline $\begin{array}{l}\text { Heroína+cocaína base mezclada en la misma } \\
\text { dosis }\end{array}$ & 67,3 & 94,4 & 98,5 & 85,3 & **** & 46,1 & 86,7 & 97,0 & 73,9 & $* * *$ \\
\hline $\begin{array}{l}\text { Heroína+cocaína polvo mezclada en la misma } \\
\text { dosis }\end{array}$ & 84,1 & 57,4 & 48,5 & 65,4 & $* * *$ & 71,4 & 43,3 & 34,5 & 52,0 & $* * *$ \\
\hline Heroína sola & 99,2 & 99,3 & 87,0 & 96,9 & & 98,4 & 95,3 & 65,0 & 90,4 & $* * *$ \\
\hline COCAÍNA & 100,0 & 100,0 & 100,0 & 100,0 & $\mathrm{~ns}$ & 94,8 & 97,7 & 99,0 & 97,5 & ns \\
\hline Cocaína base & 85,2 & 96,0 & 93,0 & 91,4 & $* * *$ & 62,0 & 89,7 & 85,0 & 78,6 & $* * *$ \\
\hline Cocaína en polvo & 97,5 & 94,6 & 86,0 & 93,9 & $* * *$ & 89,0 & 69,1 & 62,0 & 75,0 & $* * *$ \\
\hline \multicolumn{11}{|l|}{ OTRAS DROGAS } \\
\hline Ketamina & 48,1 & 10,3 & 11,0 & 24,3 & $* * *$ & 34,9 & 4,2 & 3,0 & 15,2 & $* * *$ \\
\hline Éxtasis & 83,2 & 67,9 & 49,0 & 69,8 & $* * *$ & 50,8 & 26,5 & 18,0 & 33,8 & $* * *$ \\
\hline Anfetaminas & 80,5 & 67,7 & 37,0 & 66,3 & $* * *$ & 44,1 & 16,6 & 11,0 & 25,6 & **** \\
\hline Hipnosedantes & 94,5 & 94,1 & 78,5 & 91,1 & $* * *$ & 84,3 & 86,4 & 66,0 & 81,5 & $* * *$ \\
\hline Metadona de la calle ${ }^{A}$ & 48,9 & 45,2 & 70,5 & 51,7 & $* * *$ & 32,0 & 29,7 & 57,0 & 36,1 & **** \\
\hline Opiodes diferentes a heroína o metadona & 78,0 & 76,3 & 28,5 & 67,3 & $* * *$ & 54,4 & 42,2 & 12,0 & 40,6 & *** \\
\hline Cannabis & 98,9 & 97,4 & 95,0 & 97,5 & $*$ & 93,1 & 85,7 & 87,0 & 88,7 & *** \\
\hline LSD & 81,3 & 74,9 & 50,0 & 72,3 & $* * *$ & 27,8 & 10,1 & 10,5 & 16,7 & *** \\
\hline Inhalables volátiles & 53,8 & 51,1 & 20,6 & 46,0 & **** & 10,7 & 6,6 & 4,5 & 7,7 & $*$ \\
\hline Otras & 26,6 & 14,8 & 7,5 & 17,7 & $* * *$ & & & & & \\
\hline ALCOHOL & & & & & & & & & & ns \\
\hline No consume & - & - & - & - & - & 20,1 & 16,7 & 17,1 & 18 & \\
\hline Menos de $50 \mathrm{ml}$ alcohol puro/dia & - & - & - & - & - & 49,3 & 49,4 & 46,2 & 48,7 & \\
\hline Entre 50 y $99 \mathrm{ml} /$ dia & - & - & - & - & - & 14,5 & 15,5 & 20,6 & 16,2 & \\
\hline 100 ml/día o más & - & - & - & - & - & 16,2 & 18,4 & 16,1 & 17,1 & \\
\hline TABACO & & & & & & & & & & ns \\
\hline No fumador & - & - & - & - & - & 1,9 & 2,6 & 1,0 & 2 & \\
\hline$<10$ cigarrillos/día & - & - & - & - & - & 15,9 & 13,6 & 22,5 & 16,3 & \\
\hline 10-19 cigarrarrillos/día & - & - & - & - & - & 57,1 & 56,6 & 48,0 & 55,1 & \\
\hline 20 ó más cigarrillos/día & - & - & - & - & - & 25,0 & 27,2 & 28,5 & 26,7 & \\
\hline INYECCIÓN DE DROGAS & 80,5 & 65,1 & 33,0 & 64,3 & $* * *$ & 75,8 & 48,9 & 15,5 & 52,1 & *** \\
\hline
\end{tabular}

$\$$ Nivel de significación estadística de la comparación entre ciudades, usando ji cuadrado: ns= no significativo; $*$ p <0,05; $* *$ p<0,01; *** $\mathrm{p}<0,001 ;{ }^{\mathrm{A}}$ :Se refiere a la metadona consumida fuera del marco de los programas de tratamiento.

Madrid (89,7\%) y Sevilla (85\%) que en Barcelona (62\%), y lo contrario sucedía con la cocaína-clorhidrato (cocaína polvo). Se observaron diferencias similares en el uso de mezcla de heroína-cocaína base, y de heroína-cocaína polvo, predominando la primera en Madrid y Sevilla, y la segunda en Barcelona (tabla 4).

De las restantes drogas las más extendidas eran tabaco, cannabis, alcohol, e hipnosedantes (prevalencia $>65 \%$ en últimos 12 meses), seguidas de la metadona fuera de tratamiento («metadona de la calle»), los opioides diferentes a heroína o metado- na, el éxtasis y las anfetaminas. Hubo también diferencias geográficas en el uso de estas drogas. Así, ketamina (sobre todo), éxtasis, anfetaminas y LSD estaban bastante más extendidas en Barcelona que en las otras dos ciudades. Además, en Sevilla estaba más extendida la metadona de la calle y menos los hipnosedantes y opiodes diferentes a heroína o metadona. Aproximadamente un tercio había consumido 50 $\mathrm{ml}$ de alcohol al día o más. La prevalencia de inyección reciente de drogas era muy diferente entre ciudades $(75,8 \%$ en Barcelona, $48,9 \%$ en Madrid, y 15,5\% en Sevilla) (tabla 4). 


\section{DISCUSIÓN}

En este estudio se describe la metodología y las características basales de una cohorte de jóvenes usuarios de heroína captados en Madrid, Barcelona y Sevilla. Se observa que en esta población la inyección de drogas ha dejado de ser universal, está muy extendido el uso de cocaína (frecuentemente en forma de base o crack y/o mezclada con heroína en la misma dosis), y existen importantes carencias socioeconómicas. Por otra parte, existen grandes diferencias geográficas en los patrones de uso de heroína, cocaína y otras drogas. En concreto, en Barcelona se usa sobre todo heroína-clorhidrato inyectada o esnifada, y en Madrid y Sevilla heroínabase fumada. Además, en Barcelona predomina el uso de cocaína-clorhidrato, y en Sevilla el de cocaína-base, muy a menudo mezclada con heroína. La captación de los participantes fue muy difícil.

A continuación se discuten estos hallazgos, comparándolos con los de estudios previos. Con respecto a $1995^{17}$, se observa un descenso de la inyección para administrarse drogas en las tres ciudades estudiadas. Incluso en Barcelona (donde tradicionalmente la inyección ha tenido gran relevancia), en 2001-2003 casi un 20\% de los jóvenes usuarios de heroína no se habían inyectado nunca drogas, y un 40,7\% usaban habitualmente la heroína por vías no inyectadas. En Sevilla la inyección de drogas es ya muy minoritaria, de forma que en 2001-2003 un 67\% no se habían inyectado nunca y un $99 \%$ usaban habitualmente la heroína por vía pulmonar. En Madrid, en cambio, sólo ha bajado la inyección como vía habitual de uso de heroína, y no la inyección reciente (últimos 12 meses) de drogas, lo que sugiere que los usuarios de heroína se inyectan esporádicamente heroína u otras drogas. Un análisis ulterior permitirá ahondar en este fenómeno, que, como se indicó, ha sido decisivo para el control de la infección por $\mathrm{VIH}^{11}$, y la reducción de la mortalidad por sobredosis en España $^{5}$, y, sin duda, continuará siéndolo.
Como se ha visto en estudios previos ${ }^{17}$, la vía de uso de la heroína puede cambiar a lo largo de la carrera de un consumidor, y no siempre hacia la inyección (considerada la vía más eficiente en términos de efecto/coste). De hecho, un $42,4 \%$ había cambiado alguna vez de vía principal de administración de heroína, principalmente hacia la inyección en Barcelona y hacia la vía pulmonar en Sevilla. No es fácil explicar las grandes diferencias entre Barcelona y las otras dos ciudades en la prevalencia de inyección de drogas y en la vía de uso de heroína. Sin embargo, como se ha sugerido en estudios previos $^{18}$, es muy probable que tengan que ver con el tipo de heroína circulante en el mercado. De hecho, en Madrid y Sevilla parece que circula y se usa casi exclusivamente heroína base y en Barcelona predomina la heroína-clorhidrato (que no es apta para fumar).

Otro hallazgo relevante es la gran extensión del uso de cocaína en esta población, a menudo mezclada con heroína en la misma dosis. Se observa cierta tendencia a usar la heroína y la cocaína en la misma forma química y por la misma vía. Así, en Sevilla predomina el uso de heroína y cocaína base por vía pulmonar, y en Barcelona el uso inyectado de heroína y cocaína-clorhidrato. Por otra parte, en Madrid y Sevilla la mezcla de heroína y cocaína se prepara generalmente con cocaína-base, y en Barcelona con cocaínaclorhidrato. Y, en Sevilla donde la mayoría de los participantes $(86,1 \%)$ usan habitualmente estas drogas mezcladas, el mercado ofrece una mezcla de heroína y cocaína-base ya preparada ${ }^{49,50}$. La gran extensión del uso de cocaína, y los patrones de uso adopta$\operatorname{dos}^{6,20,50}$, están condicionando seguramente los problemas sociosanitarios y las necesidades de tratamiento de estas personas. Por otra parte, además de cocaína, los jóvenes usuarios de heroína usan en altísima proporción otras drogas como tabaco, cannabis, alcohol, e hipnosedantes, lo que puede aumentar el riesgo de algunos problemas agudos de salud, sobre todo en el caso de los 
hipnosedantes y el alcohol, cuyo uso conjunto con heroína es un factor de riesgo importante de sobredosis ${ }^{5}$. De hecho, el nivel de uso de alcohol en esta población es mucho mayor que en la población general ${ }^{51}$, con una prevalencia de bebedores de riesgo (más de $50 \mathrm{ml}$ de alcohol puro/día) que supera el $30 \%$. Merece también la pena destacar el alto uso de otras drogas en Barcelona, sobre todo éxtasis, anfetaminas, ketamina (una droga que se adapta muy bien al uso inyectado), y LSD. Hay también algunas diferencias geográficas en el uso de opioides diferentes a heroína: en Sevilla el más usado es la metadona de la calle (lo que puede tener que ver con el uso de metadona en comprimidos en los programas de matenimiento ${ }^{52}$ ) y en Madrid y Barcelona otros opioides.

Los datos indican que la situación socioeconómica de los jóvenes usuarios de heroína sigue siendo muy desfavorecida. Casi la mitad no llegan a acabar la enseñanza secundaria, 1/4 carecen de vivienda estable, y más de la mitad están parados y obtienen sus mayores ingresos del robo, la venta de drogas y otras actividades marginales. Esta situación puede estar condicionando las conductas de riesgo, los problemas de salud, y las necesidades de atención de esta población ${ }^{53}$.

El mayor inconveniente de este estudio fue la dificultad para captar participantes. Aunque se hizo un gran esfuerzo (exploración de múltiples escenarios, contacto con gran número de captadores), hubo enormes dificultades para reunir la muestra prevista. Puede pensarse que estos problemas son inherentes a los estudios comunitarios con poblaciones ocultas, porque a diferencia de las cohortes clínicas, los participantes perciben que tienen poco que ganar y que revelar su identidad les puede causar problemas. Además, aunque se les gratifique, siempre cuesta desplazarse en una gran ciudad, sobre todo desde zonas donde llega mal el transporte público. Estos factores podrían explicar algunas dificultades. Sin embargo, no hubo un rechazo importante al estudio, y los problemas se centraron en encontrar candidatos seleccionables. Esto sugiere que actualmente en estas ciudades la incidencia de uso de heroína es bastante baja, lo que concuerda con los datos del Observatorio Español sobre Drogas, que indican que en España en los últimos años han descendido las admisiones a tratamiento por heroína por primera vez en la vida, las menciones de heroína en las urgencias hospitalarias, y las muertes por reacción aguda a opioides. Además, la información sobre nuevos usuarios de heroína aportada por los participantes y otras personas-clave contactados durante la captación no indica que el uso de heroína esté rebrotando, lo que no descarta que esto pueda suceder a medio o largo plazo.

Como en todos los estudios sobre poblaciones ocultas, se desconoce en qué medida se pueden generalizar los resultados al conjunto de los usuarios de heroína de las ciudades estudiadas o de España. De hecho, se utilizó un muestreo no probabilístico y se privilegió la captación de consumidores muy jóvenes o esporádicos, que suelen estar más integrados y formar redes sociales separadas de los más viejos y deteriorados, que son los más visibles en los escenarios de venta y uso masivo de drogas, y los más conocidos por captadores y servicios de atención. A pesar de esta estrategia, la proporción final de participantes de 25 años o menos $(42,1 \%)$ fue menor que la de los que superan esa edad, lo que vuelve a poner de manifiesto la falta de popularidad de la heroína entre los más jóvenes. En cualquier caso, esta cohorte incluye una proporción mayor de jóvenes y de usuarios esporádicos que algunos estudios previos sobre muestras captadas en servicios de atención o en otros lugares ${ }^{6,17}$. A pesar de ello, los resultados más relevantes relativos a los patrones de uso de drogas son bastante concordantes con dichos estudios, lo que sugiere que las importantes diferencias entre Madrid/Barcelona y Sevilla no se deben a los mecanismos de captación usados. 
Esta cohorte es quizá la más importante sobre usuarios de heroína captados en la comunidad que existe actualmente en Europa. Ofrece la posibilidad de realizar múltiples estudios transversales sobre los aspectos incluidos en el cuestionario (Anexo I). Además, aunque el seguimiento está resultando tan dificultoso como la captación, permitirá realizar estudios de incidencia o de cohortes sobre infección por VIH, mortalidad, sobredosis no mortales, etc. A finales de abril de 2005 se había logrado hacer la primera visita de seguimiento (previsto inicialmente para 12 meses después de la visita basal) a un $59,8 \%$ de los que hicieron la basal, y la segunda a un $59,3 \%$ de los que hicieron la primera en Madrid y Barcelona (sin que haya empezado esta visita en Sevilla).

\section{Anexo 1}

\section{Resumen del contenido del cuestionario basal}

\section{Características sociodemográficas}

Edad, sexo, lugar de nacimiento, migración, nivel educativo propio y de sus padres, hijos, tipo y características del alojamiento, convivientes, situación y categoría laboral, absentismo laboral, fuentes de ingresos.

\section{Conflicto social}

Fuga de casa siendo menor, detenciones, agresiones sufridas que han necesitado atención médica o psicológica, tiempo en centros de reforma, centros o familias de acogida, y cárcel.

\section{Uso de drogas psicoactivas}

- Heroína y cocaína:

- Historia de uso: edad y vía de primer uso, cambios en vía, fecha y dirección del último cambio, períodos de abstinencia.

- Consumo actual: vía, forma, lugar de consumo, tipo y cantidad consumida, uso de heroína y cocaína mezcladas y sin mezclar en la misma dosis. Para cocaína, además: sesiones intensas de consumo o "pasones", y uso de cocaína en polvo (clorhidrato de cocaína) y en base.

- Otras drogas: frecuencia de uso, y eventualmente la vía, forma de uso y cantidad consumida. Las categorías consideradas fueron: alcohol, tabaco, ketamina, éxtasis, anfetaminas, hipnosedantes, metadona de la calle, opiáceos diferentes a heroína o metadona, cannabis, LSD, inhalables volátiles y otras.

- Uso concomitante (el mismo día) de heroína e hipnosedantes.

\section{Dependencia autopercibida}

Severity Dependence Scale (SDS) para heroína y cocaína ${ }^{34,35}$.

\section{Inyección de drogas}

Circunstancias de la primera inyección, consolidación de esta vía, inyectar a otros, frecuencia de inyección subcutánea, intravenosa, e intramuscular.

\section{Inyección de riesgo}

Dar o recibir jeringas usadas, usar otro material de inyección usado antes por otros (recipientes para disolver la droga, algodones, líquido para enjuagar la jeringa), o droga preparada en dicho material.

\section{Fuentes de aprovisionamiento de jeringas estériles}

Obtención de jeringas gratuitas, compra de jeringas o de kits de inyección subvencionados

\section{Otros factores de riesgo de infección sanguínea}

Cirugía, transfusiones, tatuajes, piercing, pinchazos accidentales.

Compartir instrumentos para esnifar drogas.

\section{Conductas sexuales}

Número y tipo de parejas (estables, comerciales y ocasionales), tipo de prácticas (vaginales o anales frente a orales) y uso de preservativo. Para hombres, preguntas similares sobre relaciones homosexuales.

\section{Sobredosis por opiáceos}

- Padecidas: número, atención sanitaria, y sobre la última padecida, circunstancias, razones percibidas y uso de drogas en las horas anteriores. 
- Presenciadas: número, y sobre la mejor recordada, razones, y actuaciones de los presentes y de los servicios sanitarios.

- Sobredosis en general: precauciones para evitarlas, opiniones sobre sus causas y diversos aspectos de la atención a las mismas por los consumidores y los servicios sanitarios (ambulancias, naloxona, etc.).

Calidad de vida relacionada con la salud

Perfil de salud de Nottingham36;37.

\section{Salud Mental}

Secciones de cribado de ansiedad, estado de ánimo y psicosis de la "Entrevista Diagnostica Internacional Compuesta" (CIDI-2000)38;39, y "Cribado del International Personality Disorder Examination (IPDE) Screen" para explorar trastornos de la personalidad (eje II)

\section{Otros problemas de salud}

- Listados de síntomas y diagnósticos relacionados con uso de drogas

- Estado serológico frente a VIH: tests realizados, conocimiento de estado serológico, fechas, y, si era seropositivo, tratamiento antirretroviral

- Tests realizados y estado serológico frente a VHC, vacunación frente a VHB, diagnóstico y tratamiento de tuberculosis.

- Días en cama por problemas de salud.

- Accidentes de tráfico o de otro tipo.

Uso de servicios socio-sanitarios

- Atención en urgencias, ingresos hospitalarios, consultas médicas (medico de familia, psiquiatra, ginecólogo o tocólogo, otro)

- Atención por trabajadores sociales.

- Tratamientos de la drogodependencia (tipo de centro y tratamiento), mantenimiento con opioides (duración, opioide usado, dosis, etc.), sesiones de psicoterapia.

\section{Alimentación e higiene}

Frecuencia de lavado y ducha, frecuencia y tipo de comidas.

\section{Apoyo social}

Perfil de Duke de 8 ítems ${ }^{40 ; 41}$

Apoyo recibido ante enfermedad, necesidad de alojamiento o de dinero, y procedencia de la ayuda.

\section{Consumo de drogas por familiares y pareja}

Uso de heroína, cocaína y drogas inyectadas por padre, madre, hermanos y pareja sexual actual.

\section{AGRADECIMIENTOS}

A todas las personas e instituciones que nos han ayudado en la captación o seguimiento de participantes, que nos han prestado sus locales para la realización de las entrevistas o bien echado una mano importante en diferentes tareas de la gestión del proyecto. Entre ellos, a) En Madrid: Fundación de Ayuda contra la Drogadicción (FAD), Médicos del Mundo, Programas Municipales de Sida y de Drogas, CAID de Torrejón de Ardoz, Centro Sanitario Sandoval, Subdirección General de Sanidad de Instituciones Penitenciarias, Agencia Antidroga. b) En Barcelona: ABD, Spott, Ambit, CECAS, Secretaria de Serveis Penitenciaris, Rehabi- litació i Justícia Juvenil C) En Sevilla: Ayuntamiento de Sevilla, Centros de Tratamiento Ambulatorios de Drogodependencias de la Diputación Provincial de Sevilla, Centro Tratamiento Alcoholismo Anclaje, Antaris, Centro de Día AVAT, Asociación Aspad.

\section{BIBLIOGRAFÍA}

1. Brugal MT, Barrio G, Royuela L, Bravo MJ, de la Fuente L, Regidor E. Estimación de la mortalidad atribuible al consumo de drogas ilegales en España. Med Clin (Barc) 2004;123:775-7.

2. Sporer KA. Acute heroin overdose. Ann Intern Med 1999;130:584-90. 
3. Darke S, Zador D. Fatal heroin 'overdose': a review. Addiction. 1996;91:1765-72.

4. Darke S, Ross J, Hall W. Overdose among heroin users in Sydney, Australia: I. Prevalence and correlates of non fatal overdose. Addiction. 1996;91:405-11.

5. Brugal MT, Barrio G, De la Fuente L, Regidor E, Royuela L, Suelves JM. Factors associated with non-fatal heroin overdose: assessing the effect of frequency and route of heroin administration. Addiction. 2002;97:319-27.

6. Delegación del Gobierno para el Plan Nacional sobre Drogas. Informe $n^{\circ} 6$. Observatorio Español sobre Drogas. Noviembre 2003. Madrid: 2003.

7. Instituto de Salud Pública. Vigilancia Epidemiológica de la infección por VIH/Sida en la Comunidad de Madrid, hasta diciembre de 2003. Boletín Epidemiológico de la Comunidad de Madrid 2004;10:59-60.

8. Centro Nacional de Epidemiología y Secretaría del Plan Nacional sobre Sida. Sistema de Información sobre nuevos diagnósticos de infección por VIH (SINIVIH). 2005.

9. European Monitoring Centre for Drugs and Drug Addiction. Hepatitis $\mathrm{C}$ and injecting drug use: impact, costs and policy options. Jager L, Limbur W, Kretzschmar M, Postma M, and Wiessing L. 2004. Office for Official Publications of the European Communities.

10. Bravo MJ, Barrio G, de la Fuente L, Colomo C, Royuela L, Grupo de Trabajo de Médicos del Mundo. Persistencia de conductas de riesgo para la transmisión del VIH en inyectores de drogas de Madrid, Sevilla y Valencia. Gac Sanit 1999;13:109-18.

11. de la Fuente L, Bravo MJ, Lew C, Barrio G, Soriano V, Royuela L. Prevalencia de Infección por el virus de la inmunodeficiencia humana y de conductas de riesgo entre los consumidores de heroína de Barcelona, Madrid y Sevilla: un ejemplo de las ventajas de centrar los estudios en los consumidores y no sólo en los usuarios por vía intravenosa. Med Clin( Barc) 1999;113:646-51.

12. Fennema JS, van Ameijden EJ, Van Den HA, Coutinho RA. Young and recent-onset injecting drug users are at higher risk for HIV. Addiction. 1997;92:1457-65.

13. Van Ameijden EJC, Van den Hoek JAR, Hartgers C, Coutinho RA. Risk factors for the transition from non-injecting to injecting drug use and accompanying AIDS risk behavior in a cohort of drug users. Am J Epidemiol 1994;139:1153-63.

14. Bolumar F, Hernández-Aguado I, Ferrer L, Ruiz I, Aviñó MJ, Rebagliato M. Prevalence of antibodies to hepatitis $\mathrm{C}$ in a population of intravenous drug users in Valencia, Spain, 1990-1992. Int J Epidemiol 1996;25:204-9.

15. Garfein RS, Vlahov D, Galai N, Doherty MC, Nelson KE. Viral infections in short-term injection drug users: the prevalence of the hepatitis $\mathrm{C}$, hepatitis $\mathrm{B}$, human immunodeficiency, and human $\mathrm{T}$ lymphotropic viruses. Am J Public Health 1996;86:655-61.

16. Rodés A, Vall M, Casabona J, Nuez M, Rabella N, Mitrani L. Prevalencia de infección por el virus de la inmunodeficiencia humana y de los comportamientos asociados a su transmisión entre usuarios de drogas por vía parenteral seleccionados en la calle. Med Clín (Barc) 1998;111:372-7.

17. de la Fuente L, Barrio G, Royuela L, Bravo MJ. The transition from injecting to smoking heroin in three Spanish cities. The Spanish Group for the Study of the Route of Heroin Administration. Addiction. 1997;92:1749-63.

18. de la Fuente L, Saavedra P, Barrio G, Royuela L, Vicente J, Spanish Group for the study of the purity of seized drugs. Temporal and geographic variations in the characteristics of heroin seized in Spain and their relation with the route of administration. Drug Alcohol Depend 1996;40:185-94.

19. Des Jarlais D, Courtwright D, Joseph H. The transition from opium smoking to heroin injection in the United States. Aids Public Policy J 1991;6:88-90.

20. Barrio G, de la Fuente L, Royuela L, Díaz A, Rodríguez-Artalejo F, Spanish Group for the Study on the route of administration of drugs. Cocaine use among heroin users in Spain: the diffusion of crack and cocaine smoking. J Epidemiol Community Health 1998;52:172-80.

21. Barrio Anta G, Rodríguez Arenas MA, De La Fuente De Hoz L, Royuela L, Grupo de Trabajo para el Estudio de las Urgencias por Psicoestimulantes. Urgencias en consumidores de cocaína en varios hospitales españoles: primeras evidencias de complicaciones agudas por consumo de crack. Med Clin (Barc) 1998;111:49-55

22. Iguchi MY, Bux DA. Reduced probability of HIV infection among crack cocaine-using injection drug users. Am J Public Health 1997;87:1008-12. 
23. Krausz M, Degkwitz P, Kuhne A, Verthein U. Comorbidity of opiate dependence and mental disorders. Addict Behav 1998;23:767-83.

24. Mason BJ, Kocsis JH, Melia D, Khuri ET, Sweeney J, Wells A et al. Psychiatric comorbidity in methadone maintained patients. J Addict Dis 1998;17:7589.

25. Brugal MT, Domingo-Salvany A, Barrio G, García de Olalla P, de la Fuente L. Evaluating the impact of methadone maintenance programs on mortality due to overdose and aids in a cohort of heroin users in Barcelona city. Addiction 2005; 100: 981-989.

26. Gibson DR, Flynn N, McCarthy JJ. Effectiveness of methadone treatment in reducing HIV risk behavior and HIV seroconversion among injecting drug users. AIDS 1999;13:1807-18.

27. Drucker E, Lurie P, Wodak A, Alcabes P. Measuring harm reduction: the effects of needle and syringe exchange programs and methadone maintenance on the ecology of HIV. AIDS 1998;12:S217S230.

28. Puigdollers E, Cots F, Brugal MT, Torralba L, Domingo-Salvany A. Programas de mantenimiento con metadona con servicios auxiliares: un estudio de coste-efectividad. Gac Sanit 2003;17:12330 .

29. Torrens M, San L, Martínez A, Castillo C, Domingo-Salvany A, Alonso J. Use of the Nottingham Health Profile for measuring health status of patients in methadone maintenance treatment. Addiction. 1997;92:707-16.

30. de la Fuente L, Barrio G, Bravo MJ, Royuela L. Heroin smoking by «chasing the dragon»: its evolution in Spain. Addiction. 1998;93:444-6.

31. Watters JK, Biernacki P. Targeted sampling: options for the study of hidden populations. Social Problem 1989;36:416-30.

32. Hartnoll R, Griffiths P, Taylor C, Hendrick V, Blanken P, Nolimal D. Handbook on Snowball Sampling. Strasbourg: Pompidou Group. Council of Europe, 1997.

33. Heckathorn DD. Respondent-driven sampling: a new approach to the study of hidden populations. Soc Probl 1997;44:174-9.

34. Gossop M, Darke S, Griffiths P, Hando J, Powis B, Hall W et al. The Severity of Dependence Scale (SDS) psychometric properties of the SDS in
English and Australian samples of heroin, cocaine and amphetamines users. Addiction. 1995;90:60714.

35. Barrio G, de la Fuente L, Lew C, Royuela L, Bravo MJ, Torrens M. Differences in severity of heroin dependence by route of administration: the importance of length of heroin use. Drug Alcohol Depend 2001;63:169-77.

36. Alonso J, Antó JM, Moreno C. Spanish version of the Nottingham Health Profile: translation and preliminary validity. Am J Public Health 1990;80:7048.

37. Alonso J, Prieto C, Antó JM. The Spanish version of the Nottingham Health Profile: a review of adaptation and instrument characteristics. Qual Life Res 1994;3:385-93

38. Alonso J, Ferrer M, Romera B, Vilagut G, Angermeyer M, Bernert S et al. The European Study of the Epidemiology of Mental Disorders (ESEMeD/MHEDEA 2000) project: rationale and methods. Int J Methods Psychiatr Res 2002;11:5567.

39. Haro JM, Palacin C, Vilagut G, Romera B, Codony M, Autonell J et al. La epidemiología de los trastornos mentales en España: métodos y participación en el proyecto ESEMeD-España. Actas Esp Psiquiatr 2003;31:182-91.

40. Broadhead WE, Gehlbach SH, Degruy FV, Kaplan $\mathrm{BH}$. The Duke-UNC funcional social support questionnaire: measurement of social support in family medicine patients. Med Care 1988;26:709-23.

41. Lahuerta C, Borrel C, Rodríguez-Sanz M, Pérez K, Nebot M. La influencia de la red social en la salud mental de la población anciana. Gac Sanit 2004; 18:83-91

42. Des J, Paone D, Milliken J, Turner CF, Miller H, Gribble $\mathrm{J}$ et al. Audio-computer interviewing to measure risk behaviour for HIV among injecting drug users: a quasi-randomised trial. Lancet 1999;353:1657-61.

43. Newman JC, Des Jarlais DC, Turner CF, Gribble J, Cooley P, Paone D. The differential effects of faceto-face and computer interview modes. Am J Public Health 2002 Feb; 92(2):294-7.

44. Turner CF, Ku L, Rogers SM, Lindberg LD, Pleck JH, Sonenstein FL. Adolescent sexual behavior, drug use, and violence: increased reporting with computer survey technology. Science 1998; 280:867-73. 
45. NOVA Research Company. Questionnaire Development System 2.0 (QDSTM) . 2002. Bethesda, NOVA Research Company.

46. Secretaría del Plan Nacional del SIDA. Recomendaciones para el control de la infección por VIH, VHB y otros microorganismos de transmisión sanguínea en el medio laboral sanitario. 1994. Madrid, Ministerio de Sanidad y Consumo.

47. Bassani S, Toro C, de la Fuente L, Brugal MT, Jiménez V, Soriano V. Prevalencia de infección por virus de transmisión parenteral en consumidores actuales de heroína de 3 ciudades españolas. Med Clin.(Barc.) 2004;122:570-2

48. Darke S, Hall W, Ross M, Wodak A. Benzodiazepine use and HIV risk-taking behaviour among injecting drug users. Drug Alcohol Depend 1992;31:316.

49. Delegación del Gobierno para el Plan Nacional sobre Drogas. Plan Nacional sobre Drogas. Memoria 1996. 1997. Madrid, Ministerio de Justicia e Interior.
50. Barrio G, Bravo MJ, de la Fuente L. Consumo de drogas ilegales en España: hacia una diversificación de los patrones de consumo y los problemas asociados. Enfermedades Emergentes 2002;2:88102.

51. Consejería de Sanidad y Consumo. Consumo de alcohol y efectos sobre la salud en la población adulta y joven de la Comunidad de Madrid. Boletín Epidemiológico de la Comunidad de Madrid 2003;9:3-49.

52. Domingo-Salvany A. Los tratamientos de mantenimiento con metadona en España. Análisis de los centros activos en 1997. Junta de Andalucía. 767782. 5-11-1999. Cádiz. VI Encuentro Nacional sobre Drogodependencias y su enfoque comunitario

53. Pasarín MI, Borrell C, Brugal MT, Diaz-Quijano E. Weighing social and economic determinants related to inequalities in mortality. J Urban Health 2004;81:349-62. 